\title{
ANÁLISE COMPARATIVA DA FORCA MUSCULAR RESPIRATÓRIA DE PRIMIGESTAS NO PUERPÉRIO IMEDIATO DE PARTO NORMAL E DE NULIGESTAS
}

\section{Caroline Schwingel Wagner}

Fisioterapeuta pela Universidade de Santa Cruz do Sul (UNISC), Santa Cruz do Sul (RS), Brasil.

\section{Gabriella Assmann}

Fisioterapeuta pela Universidade de Santa Cruz do Sul (UNISC), Santa Cruz do Sul - RS, Brasil.

\section{Ricardo Gass}

Mestre em Ciências Pneumológicas pela Faculdade de Medicina da Universidade Federal do Rio Grande do Sul (UFRGS). Fisioterapeuta pela Universidade de Santa Cruz do Sul (UNISC), Santa Cruz do Sul (RS) Brasil.

\section{Marciele Silveira Hopp}

Bolsista de iniciação científica (PROBIC FAPERGS) e Fisioterapeuta pela Universidade de Santa Cruz do Sul (UNISC), Santa Cruz do Sul (RS), Brasil.

\section{Grazielly Gass Cardoso}

Bolsista de iniciação científica (PUIC voluntária) e discente do Curso de Fisioterapia da Universidade de Santa Cruz do Sul (UNISC), Santa Cruz do Sul (RS), Brasil.

\section{Dannuey Machado Cardoso}

Mestre em Ciências Médicas e doutorando em Ciências Pneumológicas pela Universidade Federal do Rio Grande do Sul (UFRGS), Porto Alegre (RS), Brasil.

\section{Dulciane Nunes Paiva}

Doutora em Ciências Médicas pela Universidade Federal do Rio Grande do Sul (UFRGS), Docente do Curso de Fisioterapia e do Programa de Pós-Graduação Stricto sensu em Promoção da Saúde da Universidade de Santa Cruz do Sul (UNISC), Santa Cruz do Sul (RS), Brasil.

\section{E-mail: dulciane@unisc.br}

RESUMO: Este artigo visa analisar a força muscular respiratória (FMR) de puérperas primíparas de parto normal e nulíparas. Trata-se de estudo do tipo caso-controle que avaliou 59 mulheres alocadas no grupo puérperas (GP) (primigestas, $n=29$ ) e no grupo controle $(\mathrm{GC})$ (nuligestas, $\mathrm{n}=30$ ). Foram avaliadas características antropométricas e FMR ( $\mathrm{PI}_{\max }$ e $\mathrm{PE}_{\max }$ ) por meio da manovacuometria digital. $\mathrm{O}$ teste $\mathrm{t}$ Student comparou dados antropométricos e da FMR entre os grupos. A correlação de Pearson $(p<0,05)$ avaliou a associação entre idade, índice de massa corporal (IMC) e FMR. Houve diferença significante na $\mathrm{PI}_{\max }(\mathrm{p}<0,001)$ e na PE ${ }_{\text {max }}(\mathrm{p}<0,001)$ entre GC e GP. A média da PI (\%predito) no GP e GC foi 39,1 $\pm 16,2 \mathrm{cmH}_{2} \mathrm{O}$ e $82,3 \pm 23,9 \mathrm{cmH}_{2} \mathrm{O}$ e a da $\mathrm{PE}_{\max }$ (\%predito) foi $34,7 \pm 14,6 \mathrm{cmH}_{2} \mathrm{O}$ e $80,4 \pm 18,4 \mathrm{cmH}_{2} \mathrm{O}$, respectivamente. Houve correlação negativa entre idade e $\mathrm{PE}_{\max }$ no $\mathrm{GP}$ $(r=-0,378 ; p=0,043)$. Evidenciou-se, sem distinção entre grupos, uma correlação negativa de pequena a moderada entre IMC e $\mathrm{PI}_{\max }$ $(\mathrm{r}=-0,380 ; \mathrm{p}=0,003)$ e $\mathrm{PE}_{\max }(\mathrm{r}=-0,525 ; \mathrm{p}<0,001)$. Foi evidenciada uma FMR menor que pode estar relacionada às alterações decorrentes da gestação na configuração da parede torácica.

PALAVRAS-CHAVE: Gravidez; Parto normal; Período Pós-Parto; Força Muscular.

\section{COMPARATIVE ANALYSIS OF RESPIRATORY MUSCLE STRENGTH IN THE IMMEDIATE PUERPERIUM OF FIRST PREGNANCY OF NATURAL CHILDBIRTH AND NULLIPAROUS}

ABSTRACT: This paper aims to analyze the respiratory muscle strength (RMS) of primiparous women of normal delivery and nulliparous. It is a case-control study that assess 59 women allocated to the Puerperal Group (PG) (primiparous, $\mathrm{n}=29$ ) and Control Group (CG) (niligestas, $n=30$ ). Anthropometric and RMS characteristics were assessed (MIP and MEP) through digital manovacuometer. The Student's t-test compared anthropometric and RMS data between groups. The Pearson correlation $(\mathrm{p}<0.05)$ assessed the association between age, body mass index (BMI) and RMS. There was a significant difference in the MIP $(p<0.001)$ and the MEP $(p<0.001)$ between CG and PG.. The average MIP (predicted\%) in PG and CG was $39.1 \pm 16.2 \mathrm{cmH}_{2} \mathrm{O}$ and $82.3 \pm 23.9 \mathrm{cmH}_{2} \mathrm{O}$ and the MEP (\% predicted) was $34.7 \pm 14.6 \mathrm{cmH}_{2} \mathrm{O}$ and $80,4 \pm 18.4 \mathrm{cmH}_{2} \mathrm{O}$, respectively. There was a negative correlation between age and MEP in PG $(r=-0.378 ; p=0.043)$. It was evidenced, without distinction between groups, a small to moderate negative correlation between BMI and MIP $(r=-0.380, p=0.003)$ and MEP $(r=$ $-0.525, \mathrm{p}<0.001)$. A lower FMR was evidenced, which may be related to changes in the chest wall configuration due to gestation. 
KEY WORDS: Pregnancy; Normal Delivery; Postpartum Period; Muscle Strength.

\section{INTRODUÇÃO}

Durante o período gestacional ocorrem intensas alterações musculoesqueléticas, físicas e emocionais que provocam mudanças fisiológicas e estruturais na gestante (BISHOP et al., 2015; SANTOS et al., 2016). O parto normal oferece diversas vantagens em relação à cesariana por tratar-se de um processo natural e não um ato cirúrgico, além de apresentar menor risco de desenvolvimento de complicações infecciosas. A taxa abusiva de parto cesáreo no Brasil que está, em média, em torno de $40 \%$, leva a mais complicações e coloca o país como líder mundial em frequência de partos cesáreos. A recomendação da Organização Mundial da Saúde (OMS, 2015) é de que a taxa ideal de cesáreas mantenha-se entre 10\% e 15\% de todos os partos.

Dentre as inúmeras alterações que ocorrem durante a gravidez, o sistema respiratório é afetado de maneira expressiva, e essas alterações persistem até o período pós-parto (COSTA et al., 2010; PINTO et al., 2015). A influência da gravidez sobre o sistema respiratório cqausa não apenas mudanças anatômicas, mas também mudanças fisiológicas que interagem e afetam a função pulmonar durante a gestação. Tais alterações podem ser consequência tanto do aumento de determinados hormônios, como a progesterona, que age estimulando o centro respiratório, promovendo o aumento da amplitude da respiração, quanto do crescimento do útero gravídico (BEZERRA; NUNES; LEMOS, 2011). As mudanças ocorrem tanto na configuração da caixa torácica, quanto nos volumes pulmonares e no deslocamento do diafragma (LEMOS et al., 2011).

Com o crescimento uterino, os músculos abdominais são submetidos a um progressivo e contínuo alongamento, podendo ocorrer aumento de até 115\% no comprimento muscular do músculo reto abdominal, tornando-o mais enfraquecido (BEZERRA; NUNES; LEMOS, 2011; CORTEZ et al., 2012). O diafragma é elevado em até $4 \mathrm{~cm}$ e, com o aumento de cerca de 2 $\mathrm{cm}$ nos diâmetros transverso e ântero-posterior do tórax, há, consequentemente, um acréscimo de 5 a 7 $\mathrm{cm}$ na circunferência da caixa torácica. $\mathrm{O}$ aumento da pressão intra-abdominal dificulta o trabalho mecânico dos músculos respiratórios por necessidade de superar, além das pressões de retração torácica e pulmonar, o aumento dessa pressão. Com o avanço da gravidez ocorre um aumento do trabalho muscular com subsequente aumento do consumo de energia (LOPES; BELAN; CASTRO, 2012).

A medida da força muscular respiratória (FMR) pode ser usada para avaliar a mecânica respiratória, bem como para quantificar a força dos músculos respiratórios (PINTO et al., 2015), sendo a manovacuometria um método considerado padrão ouro para avaliação da força muscular respiratória (LOPES; BELAN; CASTRO, 2012) (ATS/ERS, 2002). Apesar das alterações dos volumes e capacidades pulmonares e das modificações anatômicas da caixa torácica durante a gestação serem bem documentadas na literatura, ainda faz-se necessário ampliar a investigação sobre o comportamento da função pulmonar durante o puerpério imediato de parto normal (LEMOS et al., 2011). Diante do exposto, o presente estudo objetivou analisar a força muscular respiratória (FMR) de puérperas primíparas de parto normal e nulíparas hígidas.

\section{METODOLOGIA}

Trata-se de estudo observacional do tipo casocontrole que avaliou primigestas admitidas pelo Sistema Único de Saúde (SUS) na maternidade do Hospital Santa Cruz (HSC), Santa Cruz do Sul - RS, sendo a amostra não probabilística e de conveniência. Todas as pacientes assinaram o Termo de Consentimento Livre e Esclarecido (TCLE) mediante orientações sobre o protocolo proposto, tendo o presente estudo sido aprovado pelo Comitê de Ética e Pesquisa com Seres Humanos da Universidade de Santa Cruz do Sul (Unisc) e do HSC ( $\left.{ }^{\circ} 183.880\right)$.

Foram incluídas mulheres no puerpério imediato de parto vaginal no período de até 48 horas pós-parto (grupo puérpera - GP) e nuligestas com idade entre $18 \mathrm{e}$ 40 anos (grupo controle - GC). Foram excluídas aquelas com histórico de tabagismo e hipertensão arterial sistêmi- 
ca ativa, índice de massa corporal (IMC) $>40 \mathrm{~kg} / \mathrm{m}^{2}$ ou qualquer limitação cognitiva que impossibilitasse a realização dos testes, além daquelas que apresentassem labilidade emocional ou sintomas de depressão pós-parto.

\subsection{SINAIS VITAIS E CARACTERÍSTICAS ANTROPOMÉ- TRICAS}

Inicialmente foi realizada anamnese e exame físico das pacientes, sendo registrados os dados relevantes, como a história clínica, informações sobre o parto e condições clínicas do recém-nascido. Foram aferidos a pressão arterial sistólica (PAS) e diastólica (PAD) por método auscultatório (PREMIUM ${ }^{\circledR}$, modelo ESFHS50, Brasil). A freqüência respiratória (FR) foi aferida por meio da contagem do número de incursões respiratórias realizadas no período de um (01) minuto, a temperatura axilar foi aferida por meio de termômetro digital (G-Tech, EUA), enquanto a frequência cardíaca (FC) e a saturação periférica de oxigênio $\left(\mathrm{SpO}_{2}\right)$ foram aferidos por meio de oxímetro portátil de pulso (Nonim ${ }^{\circledR}$, modelo Onix 9500, Winsconsin, EUA). Todos os sinais vitais avaliados foram aferidos com o voluntário da pesquisa posicionado em decúbito dorsal no leito e em repouso, tendo sido realizadas três aferições de cada sinal vital e validado o maior valor obtido.

As características antropométricas avaliadas incluíram a estimativa do peso, altura e posterior cálculo de índice de massa corporal (IMC) por meio da fórmula de Chumlea, Roche e Steinbaugh (CHUMLEA; ROCHE; STEINBAUGH, 1985). Para tal estimativa, foram aferidas a circunferência da panturrilha (CP), a circunferência do braço (CB) e a altura do joelho (AJ) em decúbito dorsal por meio de fita antropométrica (SannyMedical ${ }^{\circledR}$ SN4010, Brasil), expressa em centímetros (cm).

A CP foi avaliada com a perna flexionada a $90^{\circ}$, tendo a fita contornado a maior circunferência da perna no sentido longitudinal. A CB foi obtida com a fita contornando o ponto médio do braço no sentido do eixo longitudinal do corpo, entre o acrômio e o olécrano. A AJ, por sua vez, foi obtida através da medida do comprimento entre o calcâneo e a cabeça da fíbula com o indivíduo mantendo a perna flexionada a $90^{\circ}$. A dobra cutânea subescapular (DCSUB) foi avaliada por meio de adipômetro (Cescorf ${ }^{\circledR}$, Brasil), tendo sido mensurada no sentido oblíquo ao eixo longitudinal do corpo, $2 \mathrm{~cm}$ abaixo do ângulo inferior da escápula (ROSA et al., 2012).

\subsection{FORÇA MUSCULAR RESPIRATÓRIA}

A FMR foi avaliada por meio da manovacuometria digital (MDI modelo MVD300, Globalmed ${ }^{\circledR}$, Porto Alegre, Brasil) que possibilitou avaliar a pressão inspiratória máxima ( $\mathrm{PI}_{\max }$ ) após expiração máxima até o volume residual, seguida de inspiração até a capacidade pulmonar total (CPT) e a pressão expiratória máxima $\left(\mathrm{PE}_{\max }\right)$, medida a partir de inspiração até a CPT, seguida por expiração até o VR, sendo ambos os testes realizados contra válvula ocluída. A medida da FMR foi realizada com o indivíduo em posição sentada em ângulo de $90^{\circ}$ com o uso de coxim de segurança na região do períneo. Preconizou-se a coleta de três medidas da FMR, com um minuto de repouso entre as mesmas, tendo sido validado o maior valor obtido, seguindo preceito da American Thoracic Society (ATS) (ATS/ERS, 2002). Os valores preditos foram baseados nos estudos de Neder et al. (1999).

\subsection{ANÁLISE ESTATÍSTICA}

Para análise dos resultados foi utilizado o software Statistical Package for Social Sciences (SPSS 23.0). A análise foi realizada mediante uma abordagem de estatística descritiva e inferencial. $\mathrm{Na}$ abordagem descritiva foi realizada a distribuição das frequências absolutas e relativas para as variáveis categóricas. A normalidade dos dados foi avaliada por meio do teste Shapiro-Wilk. Como os dados apresentaram distribuição normal, eles foram descritos em média e desvio padrão. A comparação dos dados antropométricos e da função pulmonar entre os grupos foi realizada por meio do teste $t$ Student. Para verificar a associação entre idade e IMC com a FMR, foi realizado o teste de correlação de Pearson. O nível de significância estatística considerado foi $\mathrm{p}<0,05$.

\section{RESULTADOS}

A amostra foi composta por 60 mulheres, sendo que 01 participante foi excluída por apresentar IMC $>40$ 
$\mathrm{kg} / \mathrm{m}^{2}$. A amostra final $(\mathrm{n}=59)$ foi composta por mulheres com idade média de 26,6 26,2 anos e 22,4 42,5 anos para o GC e GP, respectivamente (GP: $n=29 ; \mathrm{GC}, \mathrm{n}=30$ ). A Tabela 1 evidencia as características antropométricas e dos sinais vitais da amostra avaliada, tendo sido detectada diferença significativa entre os grupos com valores mais elevados no GP quanto ao peso $(\mathrm{p}<0,001)$, IMC $(\mathrm{p}<0,001)$, PAS $(\mathrm{p}<0,034)$ e PAD $(\mathrm{p}<0,001)$ e valores reduzidos quanto à $\mathrm{SpO}_{2}(\mathrm{p}<0,001)$ e Tax $(\mathrm{p}<0,001)$. A diferença entre as variáveis $\mathrm{PAS}, \mathrm{PAD}, \mathrm{SpO}_{2}$ e Tax não apresenta relevância clínica, pois tais variáveis encontram-se dentro dos valores de normalidade.

Tabela 1. Caracterização da amostra

\begin{tabular}{lccc}
\hline Variáveis & GP $(\mathbf{n}=\mathbf{2 9})$ & $\mathbf{G C}(\mathbf{n}=30)$ & $\boldsymbol{p}$ \\
\hline Idade (anos) & $26,6 \pm 6,2$ & $25,4 \pm 2,5$ & 0,052 \\
Peso (Kg) & $72,4 \pm 14,9$ & $59,0 \pm 7,6$ & $<0,001$ \\
Altura (m) & $1,6 \pm 0,8$ & $1,6 \pm 0,1$ & 0,920 \\
IMC $\left(\mathrm{Kg} / \mathrm{m}^{2}\right)$ & $26,6 \pm 4,7$ & $21,8 \pm 2,5$ & $<0,001$ \\
PAS $(\mathrm{mmHg})$ & $116,2 \pm 10,4$ & $110,6 \pm 9$ & 0,034 \\
PAD $(\mathrm{mmHg})$ & $73,4 \pm 8,9$ & $66 \pm 6,7$ & $<0,001$ \\
FC $(\mathrm{bpm})$ & $83,2 \pm 13,9$ & $81,7 \pm 7,9$ & 0,613 \\
SpO $(\%)$ & $95,9 \pm 1,8$ & $98,2 \pm 0,7$ & $<0,001$ \\
Tax $\left({ }^{\circ} \mathrm{C}\right)$ & $35,6 \pm 0,7$ & $36,3 \pm 0,3$ & $<0,001$ \\
\hline
\end{tabular}

GP: grupo puérpera; GC: grupo controle; IMC: índice de massa corporal; PAS: pressão arterial sistólica; PAD: pressão arterial diastólica; FC: frequência cardíaca; $\mathrm{SpO}_{2}$ : saturação periférica de oxigênio; Tax: temperatura axilar. Valores expressos em média \pm desvio padrão.

Nível de significância: $p<0,05$.

Ressalta-se que apenas no GC foram encontrados indivíduos considerados abaixo do peso (IMC $<18,50 \mathrm{~kg}$ / $\mathrm{m}^{2}$ ). Os indivíduos considerados com peso normal (IMC entre $\left.18,5-24,9 \mathrm{~kg} / \mathrm{m}^{2}\right)$ corresponderam a $37,93 \%$ no GP e $86,6 \%$ no $\mathrm{GC}$, os com sobrepeso (IMC $\geq 25 \mathrm{~kg} / \mathrm{m}^{2}$ ) classificados como pré-obesos (IMC entre 25,0 a 29,9 entre $\mathrm{kg} / \mathrm{m}^{2}$ ) representaram $37,93 \%$ do GP e $6,7 \%$ do GC. Os obesos grau I (IMC entre 30,0 a $34,9 \mathrm{~kg} / \mathrm{m}^{2}$ ) no GP foram $13,8 \%$ e os obesos grau II (IMC entre 35,0 a $39,9 \mathrm{~kg} / \mathrm{m}^{2}$ ) no GP foram 10,34\%. O GC não apresentou voluntárias com IMC classificado como obeso I, obeso II ou obeso III. No GC houve predomínio de peso normal e no GP os valores de peso normal e pré-obeso coincidiram.

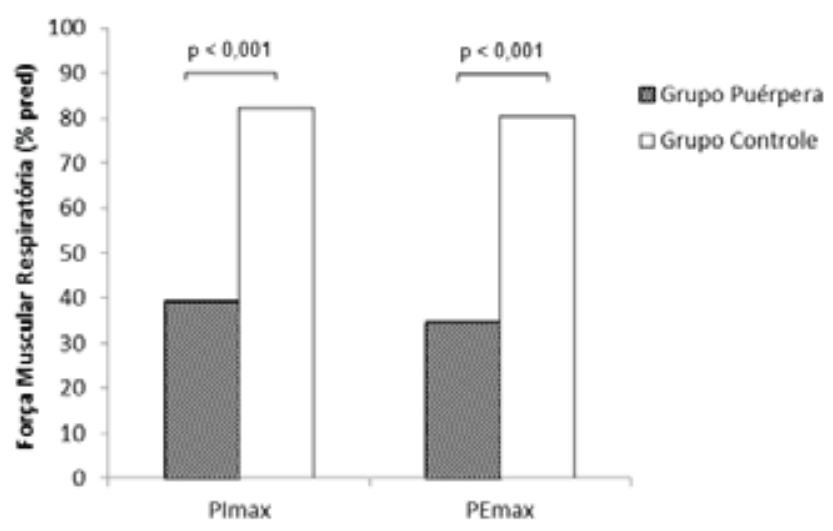

Figura 1. Análise da força muscular respiratória entre puérperas e nuligestas

Nota: Valores apresentados em média e desvio padrão. PI ${ }_{\max }$ : pressão inspiratória náxima; $\mathrm{PE}_{\max }$ : pressão expiratória máxima. Teste $t$ Student para amostras independentes $(p<0,05)$.

Como pode ser observado na Figura 1, os valores absolutos da $\mathrm{PI}_{\max }$ e da $\mathrm{PE}_{\text {max }}$ diferiram significativamente entre GP e GC $(\mathrm{p}<0,001)$, tendo o GP apresentado valores absolutos e preditos reduzidos das pressões respiratórias estáticas em relação ao GC (Tabela 2). No GP, os valores mínimos e máximos da $\mathrm{PI}_{\max }$ e da $\mathrm{PE}_{\text {max }}$ foram de - 9 a -75 $\mathrm{cmH}_{2} \mathrm{O}$ e de 21 a $78 \mathrm{cmH}_{2} \mathrm{O}$, respectivamente, e os valores mínimos e máximos de $\mathrm{PI}_{\text {max }}$ e de $\mathrm{PE}_{\text {max }}$ no GC foram de -44 a $-137 \mathrm{cmH}_{2} \mathrm{O}$ e de 57 a $142 \mathrm{cmH}_{2} \mathrm{O}$, respectivamente.

Tabela 2. Pressões respiratórias máximas da amostra avaliada

\begin{tabular}{lccc}
\hline Variáveis & $\mathbf{G P}(\mathbf{n}=\mathbf{2 9})$ & $\mathbf{G C}(\mathbf{n}=30)$ & $\boldsymbol{p}$ \\
\hline $\mathrm{PI}_{\text {max }}\left(\mathrm{cmH}_{2} \mathrm{O}\right)$ & $-38,6 \pm 16,1$ & $-83,4 \pm 24,2$ & $<0,001$ \\
$\mathrm{PI}_{\text {max }}$ (\% pred) & $39,1 \pm 16,2$ & $82,3 \pm 23,9$ & $<0,001$ \\
$\mathrm{PE}_{\text {max }}\left(\mathrm{cmH}_{2} \mathrm{O}\right)$ & $45,3 \pm 15,2$ & $91,6 \pm 19,7$ & $<0,001$ \\
$\mathrm{PE}_{\text {max }}$ (\% pred) & $34,7 \pm 14,6$ & $80,4 \pm 18,4$ & $<0,001$ \\
\hline
\end{tabular}

GP: grupo puérpera; GC: grupo controle; $\mathrm{PI}_{\max }$ : pressão inspiratória máxima; $\mathrm{PE}_{\max }$ : pressão expiratória máxima; $\%$ pred: porcentagem do predito. Valores expressos em média \pm desvio padrão. Nível de significância: $\mathrm{p}<0,05$.

No GP houve correlação negativa e significativa entre a idade das voluntárias e a $\mathrm{PE}_{\max }(\mathrm{r}=-0,378$ $\mathrm{p}=0,043$ ), o que não foi evidenciado no GC. Foi constatada, sem distinção entre os grupos, correlação negativa e significante entre o IMC e a $\mathrm{PI}_{\max }(\mathrm{r}=-0,380$; $\mathrm{p}=0,003)$ e entre o IMC e a $\mathrm{PE}_{\text {max }}(\mathrm{r}=-0,525 ; \mathrm{p}<0,001)$. Também foi constatada correlação negativa e significante entre a idade e a $\mathrm{PI}_{\text {max }}(\mathrm{r}=-0,327 ; \mathrm{p}=0,011)$ e a $\mathrm{PE}_{\text {max }}(\mathrm{r}=$ $0,435 ; \mathrm{p}=0,001)$. Deve ser ressaltado que apesar de ter 
havido correlação entre essas variáveis, tal associação foi considerada de pequena a moderada.

\section{DISCUSSÃO}

Os resultados obtidos no presente estudo evidenciaram menores valores de pressão respiratória estática do GP em relação ao GC. Durante a gestação, o crescimento uterino causa elevação do diafragma e altera de forma compensatória a configuração da caixa torácica, ocorrendo consequentemente um aumento da circunferência do tórax que nem sempre retorna ao estágio pré-gestação. Os pulmões ficam com menor área e a caixa torácica com maior diâmetro látero-lateral devido à elevação das cúpulas diafragmáticas, havendo retificação do ângulo subcostal. Com a progressão da gravidez, os músculos abdominais vão sendo submetidos a um extremo alongamento (LOPES; BELAN; CASTRO, 2012).

A mecânica ventilatória está sujeita a alterações tanto nas puérperas submetidas ao parto por via vaginal quanto às submetidas à cesariana. Durante o parto por via vaginal são encontradas evidências de fadiga diafragmática, o que pode predispor à hipocinesia muscular pós-parto e possível alteração da função pulmonar, mesmo que temporária (BEZERRA; NUNES; LEMOS, 2011; BARACHO, 2007). No presente estudo, ao comparar os valores de $\mathrm{PI}_{\text {max }}$ e $\mathrm{PE}_{\text {max }}$ das primigestas puérperas com as nuligestas, obteve-se valores significativamente menores nas puérperas.

De acordo com Costa e colaboradores (2009), puérperas de parto vaginal apresentam valores médios maiores de $\mathrm{PI}_{\text {max }}$ e $\mathrm{PE}_{\text {max }}$ que àquelas de parto cesáreo, o que pode ser explicado pela presença marcante da dor devido à incisão cirúrgica associada ao temor de realizar esforço expiratório máximo. Tanto o parto transvaginal quanto o transabdominal poder gerar prejuízos à FMR das puérperas. $O$ parto vaginal causa fadiga diafragmática devido ao grande esforço feito pela paciente, o que pode resultar na redução da $\mathrm{PI}_{\text {max }}$ (BARACHO, 2007) e na redução da capacidade ventilatória com subsequente predisposição à insuficiência respiratória. O parto cesáreo, por sua vez, pode causar ruptura do tecido subcutâneo e da aponeurose dos músculos reto abdominais o que pode prejudicar a mensuração da $\mathrm{PE}_{\max }$, devido à ação expiratória dos músculos abdominais (LEMOS et al., 2005). Já a cesárea, pode ocasionar algum grau de disfunção respiratória devido aos efeitos de anestésicos e do ato cirúrgico, o que resulta em dor e consequente diminuição da capacidade residual funcional, mesmo quando os pulmões não estejam diretamente envolvidos (COSTA et al., 2009).

Um estudo realizado em Juiz de Fora, no estado de Minas Gerais, com 24 mulheres grávidas em diferentes períodos gestacionais $\left(1^{\circ}\right.$ ao $3^{\circ}$ trimestre) evidenciou que a gestação reduziu a $\mathrm{PI}_{\max }$, quando comparada com mulheres não grávidas (LOPES; BELAN; CASTRO, 2012). Em contrapartida, há um estudo com 120 primigestas de baixo risco obstétrico, da $5^{\circ}$ à $40^{\circ}$ semana gestacional e 40 nuligestas eutróficas e sedentárias provenientes da região metropolitana do Recife - PE, no qual foi constatado que a pressão respiratória máxima das mulheres primigestas manteve-se estável durante todo o ciclo gestacional e não deferiu significativamente dos valores obtidos das nuligestas na faixa etária dos 20 aos 29 anos (LEMOS et al., 2011). No presente estudo, ao comparar a pressão respiratória com os valores de referência para a população feminina brasileira na mesma faixa etária, segundo Neder et al., (1999), evidenciou-se menor $\mathrm{PI}_{\text {max }}$ e $\mathrm{PE}_{\text {max }}$ em ambos os grupos. Tal constatação pode deve-se à idiossincrasia da amostra avaliada.

O presente estudo considerou os vários fatores que podem interferir na mensuração de pressões respiratórias máximas, podendo ser citados como limitações a variação da capacidade de entendimento do teste de força muscular estática máxima realizado pelas voluntárias e a escassez de dados na literatura sobre o comportamento da FMR em puérperas de parto normal, o que impossibilita associação com resultados expostos por outros autores. Embora os dados apresentados neste estudo tenham evidenciado que a FMR difere entre puérperas e nuligestas, tais resultados devem ser aprofundados, visto que outros fatores podem interferir no sucesso do teste. Ainda, sugere-se que as pressões respiratórias estáticas máximas sejam avaliadas durante toda a gestação e no pós-parto, com amostragem que permita estabelecer valores de referência para as puérperas e gestantes. 


\section{CONCLUSÃO}

De acordo com os dados apresentados, foi possível evidenciar que as mulheres no puerpério imediato apresentaram menor FMR em relação às não gestantes. A evidência de menor força muscular respiratória nas puérperas torna-se importante, pois pode balizar a atuação fisioterapêutica para a reabilitação pulmonar por meio de exercícios respiratórios.

\section{AGRADECIMENTO}

À Fundação de Amparo à Pesquisa do Estado do Rio Grande do Sul (FAPERGS).

\section{REFERÊNCIAS}

AMERICAN THORACIC SOCIETY/EUROPEAN RESPIRATORY SOCIETY. ATS/ERS Statement on respiratory muscle testing. Am J Respir Crit Care Med. v. 166, n. 4, p. 518-624, ago. 2002.

BARACHO, E. Fisioterapia aplicada à obstetrícia, uroginecologia e aspectos de mastologia. 4. ed. rev. e ampl. Rio de Janeiro: Guanabara Koogan, 2007.

BEZERRA, M.A.B.; NUNES, P.C.; LEMOS, A. Força muscular respiratória: comparação entre nuligestas e primigestas. Fisioter Pesq. v. 18, n. 3, p. 235-240, jul./set. 2011.

BISHOP A. et al. Current management of pregnancy-related low back pain: a national cross-sectional survey of UK physiotherapists. Physiotherapy. v. 102, n. 1, p. 78-85, mar. 2016.

CHUMLEA, W.C.; ROCHE, A.F.; STEINBAUGH, M.L. Estimating stature from knew height for persons 60 to 90 years of age. J Am Geriatr Soc. v. 33, n. 2, p. 116-20, fev. 1985.

CORTEZ, P.J et al. Correlação entre a dor lombar e as alterações posturais em gestantes. Arq Bras Ciênc Saúde. v. 37, n. 1, p. 30-35, jan./abr. 2012
COSTA, A.S.M. et al. Estudo comparativo da força muscular respiratória no puerpério imediato. Rev Bras Fisioter. v. 14, n. 1, p. 417, 2010.

COSTA, A.S.M. et al. Influência do tipo de parto na função respiratória. Teresina, PI, 2009. XIII Encontro Latino Americano de Iniciação Científica e IX Encontro Latino Americano de Pós-Graduação - Universidade do Vale do Paraíba.

LEMOS, A. et al. A avaliação da força muscular respiratório no terceiro trimestre de gestação. Rev Bras Fisioter. v. 9, n. 2, p. 151-156, mai./ago. 2005.

LEMOS, A, et al. Força muscular respiratória: comparação entre primigestas e nuligestas. J Bras Pneumol. v. 37, n. 2, p. 193-199, jan. 2011.

LOPES, G.G.; BELAN, D.F.N.; CASTRO, T.M. Análise da Pressão Inspiratória Máxima de grávidas e não grávidas.

Medicina Acadêmica. v. 1, n. 1, 2012

NEDER, J.A. et al. Reference values for lung function tests. II. Maximal respiratory pressures and voluntary ventilation. Braz J Med Biol Res, Ribeirão Preto, v. 32, n. 6, p. 19-27, jun. 1999

OMS - Organização Mundial da Saúde. Declaração da OMS sobre Taxas de Cesáreas. Organização Mundial da Saúde, 2015. Disponível em: < http://apps.who.int/iris/ bitstream/10665/161442/3/WHO_RHR_15.02_por.pdf $>$. Acesso em: 13 abr. 2017.

PINTO, A.V.A. et al. Avaliação da mecânica respiratória em gestantes. Fisioter e Pesq. v. 22, n. 4, p. 348-354. 2015.

ROSA, G. et al. Avaliação nutricional do paciente hospitalizado: uma abordagem teórico-prática, Rio de Janeiro: Guanabara, 2012.

SANTOS, M.D. et al. A dimensão da diástase abdominal tem influência sobre a dor lombar durante a gestação? Rev Dor. v. 17, n. 1, p. 43-46, jan./mar. 2016.

Recebido em: 30 de maio de 2017. Aceito em:30 de agosto de 2017 